\title{
Matrix Integrals and the Geometry of Spinors
}

\author{
Johan VAN DE LEUR \\ Mathematical Institute, Utrecht University \\ P.O. Box 80010, 3508 TA Utrecht, The Netherlands \\ E-mail:vdleur@math.uu.nl
}

Received October 9, 2000; Accepted January 10, 2001

\begin{abstract}
We obtain the collection of symmetric and symplectic matrix integrals and the collection of Pfaffian tau-functions, recently described by Peng and Adler and van Moerbeke, as specific elements in the Spin-group orbit of the vacuum vector of a fermionic Fock space. This fermionic Fock space is the same space as one constructs to obtain the $\mathrm{KP}$ and 1-Toda lattice hierarchy.
\end{abstract}

In memory of $F$ D Veldkamp

\section{Introduction}

Recently H Peng [11] showed that the symmetric matrix model and the statistics of the spectrum of symmetric matrix ensembles is governed by a strange reduction of the 2Toda lattice hierarchy [12]. Adler and van Moerbeke [4, 5] (see also [2]) show that this reduction leads to vectors of Pfaffian tau-functions. However these Pfaffian tau-functions do not satisfy the Toda lattice hierarchy, but rather another system of PDE's, which can be identified as the BKP hierarchy in the form described by V Kac and the author in [9]. To be more explicit, let $\mathcal{S}_{m}(E)$ be the set of $m \times m$ symmetric matrices with spectrum in $E \subset \mathbb{R}$ (a union of intervals), $d Z$ the Haar measure on symmetric matrices and $V(t, z)=V(z)+\sum_{i=1}^{\infty} t_{i} z^{i}$, where $V(Z)$ is a potential. Applying the spectral theorem to the symmetric matrix $Z=O^{T} \operatorname{diag}\left(z^{0}, \ldots, z_{m-1}\right) O$, with $O \in S O(m)$, we find upon integrating over the special orthogonal group the following formula for the symmetric matrix integral (see also [4]).

$$
\hat{\tau}_{m}^{E}(t)=\int_{\mathcal{S}_{m}(E)} e^{T r V(t, Z)} d Z=c_{m} \int_{\mathbb{R}^{m}}\left|\Delta_{m}(z)\right| \prod_{i=0}^{m-1}\left(e^{V\left(t, z_{i}\right)} I_{E}\left(z_{i}\right) d z_{i}\right),
$$

where $\Delta_{m}(z)=\prod_{0 \leq i<\leq j \leq m-1}\left(z_{j}-z_{i}\right)$ is the Vandermonde determinant. Adler and van Moerbeke $[4,5]$ show (see also Section 2) that these integrals for $m=2 n$ can be expressed in certain Pfaffians:

$$
\hat{\tau}_{2 n}^{E}=(2 n) ! c_{2 n} \operatorname{Pf}\left(\left(\mu_{k, \ell}(t)\right)_{0 \leq k, \ell \leq 2 n-1}\right) .
$$


Here $\operatorname{Pf}(A)$ stands for the Pfaffian of a skew-symmetric matrix $A=\left(A_{i j}\right)_{0 \leq i, j \leq 2 n-1}$ defined by

$$
\operatorname{Pf}(A)=\frac{1}{2^{n} n !} \sum_{\sigma \in S_{2 n}} \operatorname{sg}(\sigma) \prod_{j=0}^{n-1} A_{\sigma(2 j), \sigma(2 j+1)} .
$$

(if $n=0$ we assume $\operatorname{Pf}(A)=1$ ) and $\mu_{i j}(t)=\left\langle y^{i}, z^{j}\right\rangle_{t}$ are the moments of the timedependent skew-symmetric inner product

$$
\langle f, g\rangle_{t}=\iint_{E^{2}} f(y) g(z) e^{V(t, y)+V(t, z)} \operatorname{sg}(z-y) d y d z .
$$

The formula for $\hat{\tau}_{m}^{E}$ will be obtained Section 2. If $m$ is odd, it is also a Pfaffian, but the expression is more complicated.

In this paper we will show, using the Clifford algebra techniques of [9], that replacing $\hat{\tau}_{m}^{E}$ by $\tau_{m}^{E}=\frac{\hat{\tau}_{m}^{E}}{m ! c_{m}}$, the generating series,

$$
\tau^{E}(t, q)=\sum_{m=0}^{\infty} \tau_{m}^{E}(t) q^{m}
$$

of these Pfaffians is a specific element in the Spin group orbit of the vacuum vector in a fermionic Fock space. The fermionic Fock space which is constructed is more or less the same as the one one uses to obtain the KP hierarchy and 1-dim Toda lattice hierarchy. However, these tau-functions do not satisfy the KP or Toda lattice hierarchy, but we will show in section 4 that they satisfy the (charged) BKP hierarchy of [9] (see also Section 3):

$$
\begin{aligned}
\operatorname{Res} & \left(z^{n-1} e^{\xi\left(t^{\prime}, z\right)} e^{-\eta\left(t^{\prime}, z\right)} \tau_{n-1}\left(t^{\prime}\right) z^{-m-1} e^{-\xi\left(t^{\prime \prime}, z\right)} e^{\eta\left(t^{\prime \prime}, z\right)} \tau_{m+1}\left(t^{\prime \prime}\right)\right. \\
& \left.+(z)^{-n-1} e^{-\xi\left(t^{\prime}, z\right)} e^{\eta\left(t^{\prime}, z\right)} \tau_{n+1}\left(t^{\prime}\right)(z)^{m-1} e^{\xi\left(t^{\prime \prime}, z\right)} e^{-\eta\left(t^{\prime \prime}, z\right)} \tau_{m-1}\left(t^{\prime \prime}\right)\right) \\
& =\frac{1}{2}\left(1-(-1)^{n+m}\right) \tau_{n}\left(t^{\prime}\right) \tau_{m}\left(t^{\prime \prime}\right),
\end{aligned}
$$

for all $n, m \in \mathbb{Z}$, where Res $\sum f_{i} z^{i}=f_{-1}$ and

$$
\xi(t, z)=\sum_{k=1}^{\infty} t_{k} z^{k} \quad \text { and } \quad \eta(t, z)=\sum_{k=1}^{\infty} \frac{1}{k} \frac{\partial}{\partial t_{k}} z^{-k} .
$$

Note that for all $n, m \in 2 \mathbb{Z}$ these are exactly the equations for the Pfaffian tau-functions obtained by Adler and van Moerbeke in [4]. The hierarchy of equations for all $n, m \in 2 \mathbb{Z}$ is called the DKP hierarchy in [9].

In fact using the KP boson-fermion correspondence one shows that

$$
\begin{aligned}
\tau^{E}(t, q)= & \exp \left(\frac{1}{2} \iint_{E^{2}} X(t, y, z) e^{V(y)+V(z)} \operatorname{sg}(z-y) d y d z\right) \\
& \times\left(1+\int_{E} X(t, w) e^{V(w)} d w\right) \cdot 1
\end{aligned}
$$


where $X(t, z)$ and $X(t, y, z)$ are the vertex operators

$$
\begin{aligned}
X(t, z)= & q z^{q \frac{\partial}{\partial q}} \exp (\xi(t, z)) \exp (-\eta(t, z)), \\
X(t, y, z) & =X(t, z) X(t, y) \\
& =q^{2}(z-y)(y z)^{q \frac{\partial}{\partial q}} \exp (\xi(t, z)+\xi(t, y)) \exp (-\eta(t, z)-\eta(t, y)) .
\end{aligned}
$$

The symplectic matrix integrals, which can be treated in a similar way, are described in Section 8.

\section{Symmetric matrix integrals}

Let from now on $m=2 n$ if $m$ is even and $m=2 n+1$ if $m$ is odd and recall formula (1.1):

$$
\hat{\tau}_{m}^{E}(t)=c_{m} \int_{\mathbb{R}^{m}}\left|\Delta_{m}(z)\right| \prod_{i=0}^{m-1}\left(e^{V\left(t, z_{i}\right)} I_{E}\left(z_{i}\right) d z_{i}\right) .
$$

Denote by sg the sign-function and by $S_{m}$ the permutation group of $m$ letters. Using the identity

$$
\sum_{\sigma \in S_{m}} \operatorname{sg}(\sigma) \prod_{j=0}^{n-1} \operatorname{sg}(\sigma(2 j+1)-\sigma(2 j))=2^{n} n !,
$$

we find

$$
\begin{aligned}
\hat{\tau}_{m}^{E}(t)= & m ! c_{m} \int_{-\infty<z_{0}<z_{1}<\ldots<z_{m-1}<\infty} \Delta_{m}(z) \prod_{i=0}^{m-1}\left(e^{V\left(t, z_{i}\right)} I_{E}\left(z_{i}\right) d z_{i}\right) \\
= & \frac{m ! c_{m}}{2^{n} n !} \sum_{\sigma \in S_{m}} \operatorname{sg}(\sigma) \prod_{j=0}^{n-1} \operatorname{sg}\left(\sigma^{-1}(2 j+1)-\sigma^{-1}(2 j)\right) \\
& \times \int_{-\infty<z_{0}<z_{1}<\ldots<z_{m-1}<\infty} \Delta_{m}(z) \prod_{i=0}^{m-1}\left(e^{V\left(t, z_{i}\right)} I_{E}\left(z_{i}\right) d z_{i}\right) \\
= & \frac{m ! c_{m}}{2^{n} n !} \sum_{\sigma \in S_{m}} \operatorname{sg}(\sigma) \int_{-\infty<z_{0}<z_{1}<\ldots<z_{m-1}<\infty} \Delta_{m}(z) \\
& \times \prod_{j=0}^{n-1} \operatorname{sg}\left(z_{\sigma^{-1}(2 j+1)}-z_{\sigma^{-1}(2 j)}\right) \prod_{i=0}^{m-1}\left(e^{V\left(t, z_{i}\right)} I_{E}\left(z_{i}\right) d z_{i}\right) \\
= & \frac{m ! c_{m}}{2^{n} n !} \sum_{\sigma \in S_{m}} \int_{-\infty<z_{\sigma(0)}<z_{\sigma(1)}<\ldots<z_{\sigma(m-1)}<\infty} \Delta_{m}(z) \\
& \times \prod_{j=0}^{n-1} \operatorname{sg}\left(z_{2 j+1}-z_{2 j}\right) \prod_{i=0}^{m-1}\left(e^{V\left(t, z_{i}\right)} I_{E}\left(z_{i}\right) d z_{i}\right) \\
= & \frac{(m) ! c_{m}}{2^{n} n !} \int_{\mathbb{R}^{m}}^{n-1} \Delta_{m}(z) \prod_{j=0}^{n} \operatorname{sg}\left(z_{2 j+1}-z_{2 j}\right) \prod_{i=0}^{m-1}\left(e^{V\left(t, z_{i}\right)} I_{E}\left(z_{i}\right) d z_{i}\right) .
\end{aligned}
$$


If $m=2 n$ is even we continue as follows:

$$
\begin{aligned}
\hat{\tau}_{2 n}^{E}= & \frac{(2 n) ! c_{2 n}}{2^{n} n !} \sum_{\sigma \in S_{2 n}} \operatorname{sg}(\sigma) \prod_{j=0}^{n-1} \int_{\mathbb{R}^{2}}\left(z_{2 j}^{\sigma(2 j)} z_{2 j+1}^{\sigma(2 j+1)} e^{V\left(t, z_{2 j}\right)+V\left(t, z_{2 j+1}\right)}\right. \\
& \left.\times I_{E}\left(z_{2 j}\right) I_{E}\left(z_{2 j+1}\right) \operatorname{sg}\left(z_{2 j+1}-z_{2 j}\right) d z_{2 j} d z_{2 j+1}\right) \\
= & \frac{(2 n) ! c_{2 n}}{2^{n} n !} \sum_{\sigma \in S_{2 n}} \operatorname{sg}(\sigma) \prod_{j=0}^{n-1} \mu_{\sigma(2 j), \sigma(2 j+1)}(t)=(2 n) ! c_{2 n} \operatorname{Pf}\left(\left(\mu_{k, \ell}(t)\right)_{0 \leq k, \ell \leq 2 n-1}\right) .
\end{aligned}
$$

This is formula (1.2) of the introduction, with the moments $\mu_{i j}$ defined by (1.3).

If $m=2 n+1$ is odd, we use the following lemma, which can be found in Adler, Horozov and van Moerbeke [1], to continue the calculation.

Lemma 2.1. Let $A=\left(a_{i j}\right)_{0 \leq i, j \leq 2 \ell+1}$ be a skew symmetric matrix, then

$$
\operatorname{Pf}(A)=\sum_{k=0}^{2 \ell}(-)^{k} A_{k, 2 \ell+1}(\operatorname{Pf})\left(\left(A_{i j}\right)_{i, j \neq k, 2 \ell+1}\right) .
$$

Now,

$$
\begin{aligned}
\hat{\tau}_{2 n+1}^{E}(t)= & \frac{(2 n+1) ! c_{2 n+1}}{2^{n} n !} \sum_{\sigma \in S_{2 n+1}} \operatorname{sg}(\sigma) \int_{\mathbb{R}^{2 n+1}} \prod_{j=0}^{n-1} \operatorname{sg}\left(z_{2 j+1}-z_{2 j}\right) \\
& \times \prod_{i=0}^{2 n} z_{i}^{\sigma(i)}\left(e^{V\left(t, z_{i}\right)} I_{E}\left(z_{i}\right) d z_{i}\right) \\
= & \frac{(2 n+1) ! c_{2 n+1}}{2^{n} n !} \sum_{k=0}^{2 n} \sum_{\sigma \in S_{2 n+1}, \sigma(2 n)=k} \operatorname{sg}(\sigma) \int_{\mathbb{R}^{2 n+1}} z_{2 n}^{k} e^{V\left(t, z_{2 n}\right)} I_{E}\left(z_{2 n}\right) d z_{2 n} \\
& \times \prod_{j=0}^{n-1} \operatorname{sg}\left(z_{2 j+1}-z_{2 j}\right) \prod_{i=0}^{2 n-1} z_{i}^{\sigma(i)}\left(e^{V\left(t, z_{i}\right)} I_{E}\left(z_{i}\right) d z_{i}\right) \\
= & \frac{(2 n+1) ! c_{2 n+1}}{2^{n} n !} \sum_{k=0}^{2 n}(-)^{k} \sum_{\rho \in S_{2 n}^{(k)}} \operatorname{sg}(\rho) \int_{\mathbb{R}^{2 n+1}} z_{2 n}^{k} e^{V\left(t, z_{2 n}\right)} I_{E}\left(z_{2 n}\right) d z_{2 n} \\
& \times \prod_{j=0}^{n-1} \operatorname{sg}\left(z_{2 j+1}-z_{2 j}\right) \prod_{i=0}^{2 n-1} z_{i}^{\rho\left(i+\epsilon_{k}(i)\right)}\left(e^{V\left(t, z_{i}\right)} I_{E}\left(z_{i}\right) d z_{i}\right)
\end{aligned}
$$

where $\rho \in S_{2 n}^{(k)}$, the set of permutations of the numbers $1,2, \ldots, k-1, k+1, \ldots, 2 n$, is such that $\sigma(j)=\rho\left(j+\epsilon_{k}(j)\right)$, with $\epsilon_{k}(j)=0$ if $j<k$ and $=1$ if $j \geq k$. To give an idea of what we are doing, we give an example. If $n=2, k=1$ and $\sigma=\left(\begin{array}{ccccc}0 & 1 & 2 & 3 & 4 \\ 4 & 3 & 0 & 2 & 1\end{array}\right)$, then $\rho=\left(\begin{array}{cccc}0 & 2 & 3 & 4 \\ 4 & 3 & 0 & 2\end{array}\right)$ and $\operatorname{sg}(\sigma)=-\operatorname{sg}(\rho)$. Hence, 


$$
\begin{aligned}
\hat{\tau}_{2 n+1}^{E}(t)= & \frac{(2 n+1) ! c_{2 n+1}}{2^{n} n !} \sum_{k=0}^{2 n}(-)^{k} \int_{\mathbb{R}} z_{2 n}^{k}\left(e^{V\left(t, z_{2 n}\right)} I_{E}\left(z_{2 n}\right) d z_{2 n}\right) \sum_{\rho \in S_{2 n}^{(k)}} \operatorname{sg}(\rho) \\
& \times \prod_{j=0}^{n-1} \int_{\mathbb{R}^{2}}\left(z_{2 j}^{\rho\left(2 j+\epsilon_{k}(2 j)\right)} z_{2 j+1}^{\rho\left(2 j+1+\epsilon_{k}(2 j+1)\right)} e^{V\left(t, z_{2 j}\right)+V\left(t, z_{2 j+1}\right)}\right. \\
& \left.\times I_{E}\left(z_{2 j}\right) I_{E}\left(z_{2 j+1}\right) \operatorname{sg}\left(z_{2 j+1}-z_{2 j}\right) d z_{2 j} d z_{2 j+1}\right) \\
= & (2 n+1) ! c_{2 n+1} \sum_{k=0}^{2 n}(-)^{k} \int_{\mathbb{R}} z_{2 n}^{k}\left(e^{V\left(t, z_{2 n}\right)} I_{E}\left(z_{2 n}\right) d z_{2 n}\right) \\
& \times \operatorname{Pf}\left(\left(\mu_{i j}(t)\right)_{0 \leq i, j \leq 2 n, i, j \neq k}\right) \\
= & (2 n+1) ! c_{2 n+1} \sum_{k=0}^{2 n}(-)^{k} \mu_{k}(t) \operatorname{Pf}\left(\left(\mu_{i j}(t)\right)_{0 \leq i, j \leq 2 n, i, j \neq k}\right) \\
= & (2 n+1) ! c_{2 n+1} \operatorname{Pf}\left(M_{2 n+1}(t)\right),
\end{aligned}
$$

where

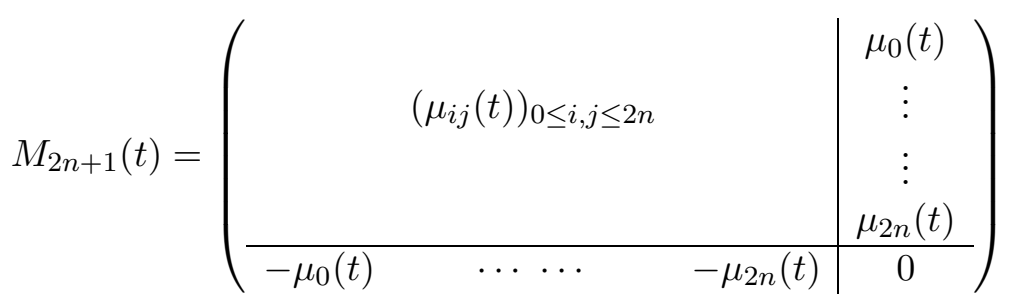

and $\mu_{i}(t)=\left(1, z^{i}\right)_{t}$ are the moments of the time-dependent symmetric inner product

$$
(f, g)_{t}=\int_{E} f(z) g(z) e^{V(t, z)} d z .
$$

The symplectic matrix integrals, which can be treated in a similar way, are described in Section 8 .

\section{The geometry of spinors and the BKP hierarchy}

In this section we recall the results of [9].

Consider the vector space $V=V^{+} \oplus V^{0} \oplus V^{-}$, where $V^{ \pm}=\underset{i \in \mathbb{Z}+\frac{1}{2}}{\bigoplus} \mathbb{C} \psi_{i}^{ \pm}$and $V^{0}=\mathbb{C} \psi_{0}$ with symmetric bilinear form

$$
\left(\psi_{0}, \psi_{0}\right)=1, \quad\left(\psi_{0}, \psi_{i}^{ \pm}\right)=0, \quad\left(\psi_{i}^{ \pm}, \psi_{j}^{ \pm}\right)=0, \quad\left(\psi_{i}^{ \pm}, \psi_{j}^{\mp}\right)=\delta_{i,-j} .
$$

Let $C \ell V$ be the associated Clifford algebra, that is the quotient of the tensor algebra over $V$ by the ideal generated by relations

$$
u v+v u=(u, v) 1, \quad \text { where } u, v \in V .
$$

These relations induce a natural $\mathbb{Z} / 2 \mathbb{Z}$ decompostion $C \ell V=C \ell V_{\overline{0}} \oplus C \ell V_{\overline{1}}$. Denote by $(C \ell V)^{\times}$the multiplicative group of invertible elements of the algebra $C \ell V$, by Pin $V$ 
the subgroup of $(C \ell V)^{\times}$generated by all the elements $a$ such that $a V a^{-1}=V$ and let Spin $V=$ Pin $V \cap C \ell_{0} V$. A simple but for this paper important observation is that for $\lambda=+,-$, both $1+\sum_{-N \leq i \leq M} c_{i} \psi_{0} \psi_{i}^{\lambda} \in \operatorname{Spin} V$ and

$$
\exp \left(\sum_{-N \leq i<j \leq M} c_{i j} \psi_{i}^{\lambda} \psi_{j}^{\lambda}\right) \in \operatorname{Spin} V .
$$

We have a homomorphism $T: \operatorname{Pin} V \rightarrow O(V), g \mapsto T_{g}$ defined by $(v \in V)$ :

$$
T_{g}(v)=g v g^{-1} \in V \text {. }
$$

Denote by $U=\sum_{j>0}\left(\mathbb{C} \psi_{j}^{+}+\mathbb{C} \psi_{j}^{-}\right)+\mathbb{C}\left(1+\sqrt{2} \psi_{0}\right)$ the subspace of $\mathbb{C} 1+V$ and let

$$
F:=F(V, U)=C \ell V /(C \ell V) U \text {. }
$$

The space $F(V, U)$ caries a structure of a $C \ell V$-module induced by left multiplication. This module restricted to Pin $V$ is called the spin module of the group Pin $V$. This module remains irreducible when restricted to Spin $V$. Denote the image of 1 in $F(V, U)$ by $|0\rangle$, then

$$
\psi_{j}^{ \pm}|0\rangle=0 \quad \text { if } j>0 \quad \text { and } \quad \psi_{0}|0\rangle=-\frac{1}{\sqrt{2}}|0\rangle .
$$

By introducing the notion of charge as follows:

$$
\text { charge }|0\rangle=\text { charge } \psi_{0}=0, \quad \text { charge } \psi_{j}^{ \pm}= \pm 1,
$$

the space $F$ decomposes into charge sectors

$$
F=\bigoplus_{k \in \mathbb{Z}} F_{k}
$$

Given $f \in F$, let

$$
\text { Ann } f=\{v \in V \mid v f=0\} \text {, }
$$

then the vacuum vector $|0\rangle \in F$ is characterized (up to a constant factor) among the vectors of $F$ by the property that

$$
\text { Ann }|0\rangle=U_{0}:=\sum_{j>0}\left(\mathbb{C} \psi_{j}^{+}+\mathbb{C} \psi_{j}^{-}\right) .
$$

Moreover, let $g \in$ Pin $V$, then

$$
\text { Ann } g|0\rangle=T_{g}(\text { Ann }|0\rangle)=T_{g}\left(U_{0}\right) .
$$

All maximal isotropic subspaces of $V$ characterize the Spin $V$-group orbit. Let $O=$ Spin $V \cdot|0\rangle$ be the Spin $V$-orbit of $|0\rangle$, one of the main observations of the paper [9] is the following: 
Proposition 3.1. If $\tau \in F$ and $\tau \neq 0$, then $\tau \in O$ if and only if $\tau$ satisfies the equation

$$
\psi_{0} \tau \otimes \psi_{0} \tau+\sum_{j \in \mathbb{Z}+\frac{1}{2}}\left(\psi_{j}^{+} \tau \otimes \psi_{-j}^{-} \tau+\psi_{j}^{-} \tau \otimes \psi_{-j}^{+} \tau\right)=\frac{1}{2} \tau \otimes \tau .
$$

This equation is called the fermionic BKP hierarchy. We will now rewrite these equations to a hierarchy of differential equations. For this we use the classical boson-fermion correspondence [10]. Consider the following generating series, called charged fermionic fields:

$$
\psi^{ \pm}(z)=\sum_{i \in \frac{1}{2}+\mathbb{Z}} \psi_{i}^{ \pm} z^{-i-\frac{1}{2}}
$$

Then we have:

$$
\psi^{\lambda}(y) \psi^{\mu}(z)+\psi^{\mu}(z) \psi^{\lambda}(y)=\delta_{\lambda,-\mu} \delta(y-z), \quad \lambda, \mu= \pm,
$$

where

$$
\delta(y-z)=y^{-1} \sum_{n \in \mathbb{Z}}\left(\frac{y}{z}\right)^{n} .
$$

We split up field $\phi(z)=\sum_{i} \phi_{i} z^{i}$ in its positive and negative part:

$$
\phi(z)=\phi(z)_{+}+\phi(z)_{-}
$$

where

$$
\phi_{+}=\sum_{i \geq 0} \phi_{i} z^{i} \quad \text { and } \quad \phi(z)_{-}=\phi(z)-\phi(z)_{+}
$$

Define the bosonic fields $(\nu \in \mathbb{C})$

$$
\begin{aligned}
& \alpha(z)=\sum_{k \in \mathbb{Z}} \alpha_{k} z^{-k-1}=: \psi^{+}(z) \psi^{-}(z): \\
& L^{\nu}(z)=\sum_{k \in \mathbb{Z}} L_{k}^{\nu} z^{-k-2}=\frac{1}{2}: \alpha(z) \alpha(z):+\left(\frac{1}{2}-\nu\right) \partial_{z}(\alpha(z)), \\
& Y^{ \pm}(z)=\sum_{k \in \mathbb{Z}} Y_{k}^{ \pm} z^{-k-2}=\partial_{z}\left(\psi^{ \pm}(z)\right) \psi^{ \pm}(z),
\end{aligned}
$$

where the normally ordered product of two fields is defined, as usual, by

$$
: \phi(y) \rho(z):=\phi(y)_{+} \rho(z)-\rho(z) \phi(y)_{-} .
$$


Then one has (using Wick's formula) $(\lambda= \pm$ ):

$$
\begin{aligned}
& {\left[\alpha_{k}, \psi^{\lambda}(z),\right]=\lambda z^{k} \psi^{\lambda}(z),} \\
& {\left[\alpha_{k}, Y^{\lambda}(z),\right]=2 \lambda z^{k} Y^{\lambda}(z),} \\
& {\left[L_{k}^{\nu}, \psi^{\lambda}(z)\right]=\left(z^{k+1} \partial_{z}+\left(\lambda\left(\nu-\frac{1}{2}\right)+\frac{1}{2}\right)(k+1) z^{k}\right) \psi^{\lambda}(z),} \\
& {\left[L_{k}^{\nu}, Y^{\lambda}(z)\right]=\left(z^{k+1} \partial_{z}+\left(2 \lambda\left(\nu-\frac{1}{2}\right)+2\right)(k+1) z^{k}\right) Y^{\lambda}(z),} \\
& {\left[L_{k}^{\nu}, \alpha(z)\right]=\left(z^{k+1} \partial_{z}+(k+1) z^{k}\right) \alpha(z)+\left(\nu-\frac{1}{2}\right)(k+1) k z^{k-1},} \\
& {\left[\alpha_{k}, \alpha(z)\right]=k z^{k-1},} \\
& {\left[L_{k}^{\nu}, L_{\ell}^{\nu}\right]=(k-\ell) L_{k+\ell}^{\nu}+\delta_{k,-\ell} \frac{k^{3}-k}{12} c_{\nu},}
\end{aligned}
$$

where $c_{\nu}=-12 \nu^{2}+12 \nu-2$ and $\psi_{0}$ commutes with all these bosonic operators.

Thus, the $\alpha_{k}, L_{k}^{\nu}$ form the oscillator algebra, respectively Virasoro algebra and

$$
\begin{array}{ll}
\alpha_{k}|0\rangle=0 & \text { for } \quad k \geq 0, \\
L_{k}^{\nu}|0\rangle=0 & \text { for } k \geq-1, \\
Y_{k}^{ \pm}|0\rangle=0 & \text { for } \quad k \geq 0 .
\end{array}
$$

The operator $\alpha_{0}$ is diagonalizable in $F$, with eigenspaces the charge sectors $F_{k}$, i.e. $\alpha_{0} f_{k}=k f_{k}$ for $f_{k} \in F_{k}$. For that reason we call $\alpha_{0}$ the charge operator.

In order to express the fermionic fields $\psi^{\lambda}(z)$ in terms of the oscillator algebra, we need an additional operator $Q$ on $F$ defined by

$$
Q|0\rangle=\psi_{-\frac{1}{2}}^{+}|0\rangle, \quad Q \psi_{k}^{+}=\psi_{k \mp 1}^{ \pm} Q, \quad Q \psi_{0}=-\psi_{0} Q .
$$

\section{Proposition 3.2. $([6,7,8])$}

$$
\begin{aligned}
& \psi^{ \pm}(z)=Q^{ \pm 1} z^{ \pm \alpha_{0}} \exp \left(\mp \sum_{k<0} \frac{\alpha_{k}}{k} z^{-k}\right) \exp \left(\mp \sum_{k>0} \frac{\alpha_{k}}{k} z^{-k}\right), \\
& Y^{ \pm}(z)=Q^{ \pm 2} z^{ \pm 2 \alpha_{0}} \exp \left(\mp 2 \sum_{k<0} \frac{\alpha_{k}}{k} z^{-k}\right) \exp \left(\mp 2 \sum_{k>0} \frac{\alpha_{k}}{k} z^{-k}\right) .
\end{aligned}
$$

We now identify $F$ with the space $B=\mathbb{C}\left[q, q^{-1}, t_{1}, t_{2}, \ldots\right]$ via the vector space homorphism

$$
\sigma: F \stackrel{\sim}{\rightarrow} B
$$

given by

$$
\sigma\left(\alpha_{-m_{1}} \ldots \alpha_{-m_{s}} Q^{k}|0\rangle\right)=m_{1} m_{2} \ldots m_{s} t_{m_{1}} t_{m_{2}} \ldots t_{m_{s}} q^{k} .
$$

The transported charge is as follows

$$
\text { charge }\left(p(t) q^{k}\right)=k \text {, }
$$


and the transported charge decomposition is

$$
B=\bigoplus_{m \in \mathbb{Z}} B_{m}, \quad \text { where } \quad B_{m}=\mathbb{C}\left[t_{1}, t_{2} \ldots\right] q^{m} .
$$

The transported operators $\psi_{0}, \alpha_{m}$ and $Q$ on $B$ are as follows:

$$
\begin{array}{ll}
\sigma \psi_{0} \sigma^{-1}=-\frac{1}{\sqrt{2}}(-)^{q} \frac{\partial}{\partial q}, & W_{-m}=\sigma \alpha_{-m} \sigma^{-1}=m t_{m}, \\
W_{m}=\sigma \alpha_{m} \sigma^{-1}=\frac{\partial}{\partial t_{m}}, & W_{0}=\sigma \alpha_{0} \sigma^{-1}=q \frac{\partial}{\partial q}, \\
\sigma Q \sigma^{-1}=q . &
\end{array}
$$

Hence

$$
\begin{aligned}
& \sigma \psi^{ \pm}(z) \sigma^{-1}=q^{ \pm 1} z^{ \pm q} \frac{\partial}{\partial q} e^{ \pm \xi(t, z)} e^{\mp \eta(t, z)}, \\
& \sigma Y^{ \pm}(z) \sigma^{-1}=q^{ \pm 2} z^{ \pm 2 q \frac{\partial}{\partial q}} e^{ \pm 2 \xi(t, z)} e^{\mp 2 \eta(t, z)},
\end{aligned}
$$

where $\xi(t, z)$ and $\eta(t, z)$ are defined in (1.5). It is now straightforward to prove the following

Lemma 3.1. For $y>z$ one has

$$
\begin{aligned}
& \sigma \psi^{\lambda}(y) \psi^{\mu}(z) \sigma^{-1}=(y-z)^{\lambda \mu 1} q^{\lambda+\mu} y^{\lambda q \frac{\partial}{\partial q}} z^{\mu q \frac{\partial}{\partial q}} e^{\lambda \xi(t, y)+\mu \xi(t, z)} e^{-\lambda \eta(t, y)-\mu \eta(t, z)}, \\
& \sigma Y^{\lambda}(y) Y^{\mu}(z) \sigma^{-1}=(y-z)^{\lambda \mu 4} q^{\lambda 2+\mu 2} y^{\lambda 2 q \frac{\partial}{\partial q}} z^{\mu 2 q \frac{\partial}{\partial q}} e^{\lambda 2 \xi(t, y)+\mu 2 \xi(t, z)} e^{-\lambda 2 \eta(t, y)-\mu 2 \eta(t, z)} .
\end{aligned}
$$

Using the commutation relations (3.6) one immediately obtains the following consequence of this lemma:

Corollary 3.1. For $z_{0}, z_{1}, \ldots, z_{m} \in \mathbb{R}$ distinct we have

$$
\begin{gathered}
\sigma \psi^{+}\left(z_{m}\right) \psi^{+}\left(z_{m-1}\right) \cdots \psi^{+}\left(z_{1}\right) \psi^{+}\left(z_{0}\right) \sigma^{-1} \\
=q^{m+1} \Delta_{m+1}(z) \prod_{i=0}^{m} z_{i}^{q} \frac{\partial}{\partial q} e^{\sum_{j=0}^{m} \xi\left(t, z_{j}\right)} e^{-\sum_{j=0}^{m} \eta\left(t, z_{j}\right)}, \\
\sigma Y^{+}\left(z_{m}\right) Y^{+}\left(z_{m-1}\right) \cdots Y^{+}\left(z_{1}\right) Y^{+}\left(z_{0}\right) \sigma^{-1} \\
=q^{2 m+2} \Delta_{m+1}^{4}(z) \prod_{i=0}^{m} z_{i}^{2 q \frac{\partial}{\partial q}} e^{\sum_{j=0}^{m} 2 \xi\left(t, z_{j}\right)} e^{-\sum_{j=0}^{m} 2 \eta\left(t, z_{j}\right)} .
\end{gathered}
$$

We will now use the boson-fermion correspondence to rewrite the BKP hierarchy. Notice first that (3.4) is equivalent to

$$
\operatorname{Res}\left(\psi^{+}(z) \tau \otimes \psi^{-}(z) \tau+\psi^{-}(z) \tau \otimes \psi^{+}(z) \tau\right)=\frac{1}{2}\left(\tau \otimes \tau-\psi_{0} \tau \otimes \psi_{0} \tau\right),
$$

where $\operatorname{Res} \sum f_{i} z^{i}=f_{-1}$. Now apply $\sigma$ to (3.11). Writing $\sigma(\tau)=\sum_{n \in \mathbb{Z}} \tau_{n}(t) q^{n}$, we obtain for all $n, m \in \mathbb{Z}$ equation (1.4) as the coefficient of $q^{n} \otimes q^{m}$.

The Spin group elements which we consider in the rest of the paper do not fit in the algebraic framework of this section. However, we will need them to describe the symmetric and symplectic matrix integrals as (generalized) tau-functions. Since all manipulations with vertex operators, used there, are well defined and correct, it is clear what we mean and we are doing. 


\section{Pfaffian tau-functions}

Let $F(z)$ (resp. $F(y, z))$ be a (skew-symmetric) weight function on $\mathbb{R}\left(\mathbb{R}^{2}\right)$ and let

$$
\begin{aligned}
(f, g) & =\int_{\mathbb{R}} f(z) g(z) F(z) d z, \quad \text { respectively } \\
\langle f, g\rangle & =\iint_{\mathbb{R}^{2}} f(y) g(z) F(y, z) d y d z
\end{aligned}
$$

be the corresponding symmetric (skew-symmetric) inner product. Consider the following deformation of these inner products, which we assume to be the time-dependent $t=$ $\left(t_{1}, t_{2}, \cdots\right)$ :

$$
\begin{aligned}
(f, g)_{t} & =\int_{\mathbb{R}} f(z) g(z) e^{\xi(t, z)} F(z) d z, \\
\langle f, g\rangle_{t} & =\iint_{\mathbb{R}^{2}} f(y) g(z) e^{\xi(t, y)+\xi(t, z)} F(y, z) d y d z,
\end{aligned}
$$

with $\xi(t, z)=\sum_{i} t_{i} z^{i}$ as before. Notice that the inner products appearing in Section 1 and 2 are special cases of (4.2). Let

$$
\begin{aligned}
& \mu_{i}(t)=\left(1, z^{i}\right)_{t} \text { and } \mu_{i}=\mu_{i}(0)=\left(1, z^{i}\right), \\
& \mu_{i j}(t)=\left\langle y^{i}, z^{j}\right\rangle_{t}=-\mu_{j i}(t) \text { and } \mu_{i j}=\mu_{i j}(0)=\left\langle y^{i}, z^{j}\right\rangle=-\mu_{j i}
\end{aligned}
$$

be the moments. Consider the following generalized Spin group element (see Section 3):

$$
G^{\mu}=\exp \left(g^{\mu}\right) \cdot f^{\mu}=\exp \left(\sum_{-\infty<j<i<0} \mu_{-i-\frac{1}{2},-j-\frac{1}{2}} \psi_{j}^{+} \psi_{i}^{+}\right)\left(1+\sqrt{2} \sum_{k<0} \mu_{-k-\frac{1}{2}} \psi_{0} \psi_{k}^{+}\right) .
$$

We calculate its action on the vacuum vector $|0\rangle$, let

$$
\begin{aligned}
\tau^{F}= & \exp \left(\sum_{-\infty<j<i<0} \mu_{-i-\frac{1}{2},-j-\frac{1}{2}} \psi_{j}^{+} \psi_{i}^{+}\right)\left(1+\sqrt{2} \sum_{k<0} \mu_{-k-\frac{1}{2}} \psi_{0} \psi_{k}^{+}\right)|0\rangle \\
= & \exp \left(\frac{1}{2} \sum_{i, j<0} \mu_{-i-\frac{1}{2},-j-\frac{1}{2}} \psi_{j}^{+} \psi_{i}^{+}\right)\left(1+\sum_{k<0} \mu_{-k-\frac{1}{2}} \psi_{k}^{+}\right)|0\rangle \\
= & \sum_{n=0}^{\infty} \frac{1}{2^{n} n !}\left(\sum_{i, j<0} \mu_{-i-\frac{1}{2},-j-\frac{1}{2}} \psi_{j}^{+} \psi_{i}^{+}\right)^{n}\left(1+\sum_{k<0} \mu_{-k-\frac{1}{2}} \psi_{k}^{+}\right)|0\rangle \\
= & \sum_{n=0}^{\infty} \frac{1}{2^{n} n !}\left(\sum_{i, j<0} \iint_{\mathbb{R}^{2}} y^{-i-\frac{1}{2}} z^{-j-\frac{1}{2}} F(y, z) d y d z \psi_{j}^{+} \psi_{i}^{+}\right)^{n} \\
& \times\left(1+\int_{\mathbb{R}} \sum_{k<0} w^{-k-\frac{1}{2}} \psi_{k}^{+} F(w) d w\right)|0\rangle
\end{aligned}
$$




$$
\begin{aligned}
= & \sum_{n=0}^{\infty} \frac{1}{2^{n} n !}\left(\iint_{\mathbb{R}^{2}} \psi^{+}(z) \psi^{+}(y) F(y, z) d y d z\right)^{n}\left(1+\int_{\mathbb{R}} \psi^{+}(w) F(w) d w\right)|0\rangle \\
= & \sum_{n=0}^{\infty} \frac{1}{2^{n} n !}\left(\int_{\mathbb{R}^{2 n}} \psi^{+}\left(z_{2 n-1}\right) \psi^{+}\left(z_{2 n-2}\right) \cdots \psi^{+}\left(z_{0}\right) \prod_{j=0}^{n-1} F\left(z_{2 j}, z_{2 j+1}\right) d z_{2 j} d z_{2 j+1}\right) \\
& \quad \times\left(1+\int_{\mathbb{R}} \psi^{+}\left(z_{2 n}\right) F\left(z_{2 n}\right) d z_{2 n}\right)|0\rangle \\
= & \sum_{m=0}^{\infty} \tau_{m}^{F} .
\end{aligned}
$$

Using the boson-fermion correspondence and corollary 3.1 we rewrite this as follows, let $\tau^{F}(t, q)=\sigma\left(\tau^{F}\right)$, then

$$
\begin{aligned}
\tau^{F}(t, q)=\sum_{n=0}^{\infty} & \frac{1}{2^{n} n !}\left(\int_{\mathbb{R}^{2 n}} \Delta_{2 n}(z) \prod_{j=0}^{n-1} e^{\xi\left(t, z_{2 j}\right)+\xi\left(t, z_{2 j+1}\right)} F\left(z_{2 j}, z_{2 j+1}\right) d z_{2 j} d z_{2 j+1}\right) q^{2 n} \\
& +\sum_{n=0}^{\infty} \frac{1}{2^{n} n !}\left(\int_{\mathbb{R}^{2 n+1}} \Delta_{2 n+1}(z) e^{\xi\left(t, z_{2 n}\right)} F\left(z_{2 n}\right) d z_{2 n}\right. \\
& \left.\times \prod_{j=0}^{n-1} e^{\xi\left(t, z_{2 j}\right)+\xi\left(t, z_{2 j+1}\right)} F\left(z_{2 j}, z_{2 j+1}\right) d z_{2 j} d z_{2 j+1}\right) q^{2 n+1} \\
= & \sum_{m=0}^{\infty} \tau_{m}^{F}(t) q^{m} .
\end{aligned}
$$

Then $\tau_{0}^{F}(t)=1$,

$$
\begin{aligned}
\tau_{2 n}^{F}(t)= & \frac{1}{2^{n} n !} \int_{\mathbb{R}^{2 n}} \sum_{\sigma \in S_{2 n}} \operatorname{sg}(\sigma) z_{0}^{\sigma(0)} z_{1}^{\sigma(1)} \cdots z_{2 n-1}^{\sigma(2 n-1)} \\
& \times \prod_{j=0}^{n-1} e^{\xi\left(t, z_{2 j}\right)+\xi\left(t, z_{2 j+1}\right)} F\left(z_{2 j}, z_{2 j+1}\right) d z_{2 j} d z_{2 j+1} \\
= & \frac{1}{2^{n} n !} \sum_{\sigma \in S_{2 n}} \operatorname{sg}(\sigma) \prod_{j=0}^{n-1} \mu_{\sigma(2 j), \sigma(2 j+1)}(t) \\
= & \operatorname{Pf}\left(\left(\mu_{k, \ell}(t)\right)_{0 \leq k, \ell \leq 2 n-1}\right)
\end{aligned}
$$

and

$$
\begin{aligned}
\tau_{2 n+1}^{E} F(t)=\frac{1}{2^{n} n !} & \int_{\mathbb{R}^{2 n+1}} \sum_{\sigma \in S_{2 n+1}} \operatorname{sg}(\sigma) z_{0}^{\sigma(0)} z_{1}^{\sigma(1)} \cdots z_{2 n}^{\sigma(2 n)} F\left(z_{2 n}\right) \\
& \times \prod_{j=0}^{n-1} F\left(z_{2 j}, z_{2 j+1}\right) \prod_{i=0}^{2 n} e^{\xi\left(t, z_{i}\right)} d z_{i}
\end{aligned}
$$


A similar calculation as in (2.3) and (2.4) now shows that $\tau_{2 n+1}^{F}(t)=\operatorname{Pf}\left(M_{2 n+1}(t)\right)$, where $M_{2 n+1}(t)$ is given by (2.5). Hence we have shown:

Theorem 4.1. Let $\mu_{i}(t), \mu_{i j}(t)$ be the moments of the symmetric, respectively skewsymmetric innerproduct $\left.(\cdot, \cdot)_{t}\right)$ and $\langle\cdot, \cdot\rangle_{t}$, defined by (4.2). Let $M_{2 n}(t)=\left(\mu_{i j}(t)\right)_{0 \leq i, j \leq 2 n-1}$ and

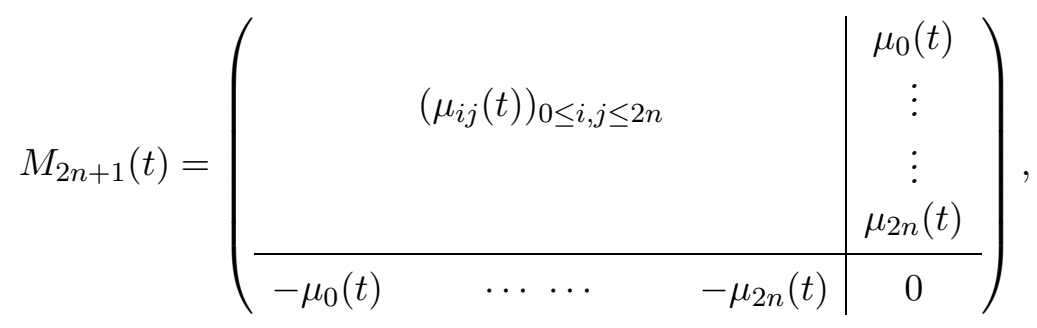

then

$$
\begin{gathered}
\sigma\left(\exp \left(\sum_{-\infty<j<i<0} \mu_{-i-\frac{1}{2},-j-\frac{1}{2}}(0) \psi_{j}^{+} \psi_{i}^{+}\right)\left(1+\sqrt{2} \sum_{k<0} \mu_{-k-\frac{1}{2}}(0) \psi_{0} \psi_{k}^{+}\right)|0\rangle\right) \\
=\exp \left(\frac{1}{2} \iint_{\mathbb{R}^{2}} X(t, y, z) F(y, z) d y d z\right)\left(1+\int_{\mathbb{R}} X(t, w) F(w) d w\right) \cdot 1 \\
=\sum_{m=0}^{\infty} \operatorname{Pf}\left(M_{m}\right)(t) q^{m}
\end{gathered}
$$

where $X(t, y, z)$ and $X(t, w)$ are given by (1.6).

Since $\tau^{F}$ is an element in the spin group orbit of the vacuum vector, one has the following consequence:

Corollary 4.1. The Pfaffian tau-functions $\tau_{m}^{F}(t)=\operatorname{Pf}\left(M_{m}(t)\right), m \in \mathbb{Z}$, satisfy the BKP hierarchy (1.4).

Since $f^{\mu}$ and $\exp \left(g^{\mu}\right)$ commute, we find that

$$
\tau_{2 n+1}^{F}=\sqrt{2} \sum_{k<0} \mu_{-k-\frac{1}{2}} \psi_{0} \psi_{k}^{+} \tau_{2 n}^{F}
$$

Hence,

$$
\tau_{2 n+1}^{F}(t)=\int_{\mathbb{R}} w^{2 n} e^{\xi(t, w)} e^{-\eta(t, w)} \tau_{2 n}^{F}(t) F(w) d w
$$

and $\tau_{2 n+1}^{F}(t)$ is completely determined by $\tau_{2 n}^{F}(t)$

\section{Virasoro constraints}

In this section we give a representation theoretical proof of the Virasoro constraints for the symmetric matrix integrals obtained by Adler and van Moerbeke in [4]. Our proof is along the lines of [3]. 
As before, consider the matrix integral over symmetric matrices

$$
\hat{\tau}_{m}^{E}(t)=m ! c_{m} \tau_{m}^{E}(t)=\int_{\mathcal{S}_{m}(E)} e^{\operatorname{Tr}\left(V(Z)+\sum_{1}^{\infty} t_{i} Z^{i}\right)} d Z,
$$

integrated over the space $\mathcal{S}_{m}(E)$ of symmetric matrices with spectrum in $E \subset \mathbb{R}$, where

$$
E=\operatorname{disjoint} \text { union } \bigcup_{i=1}^{r}\left[c_{2 i-1}, c_{2 i}\right]
$$

and we assume that the potential $V$ satisfies

$$
V^{\prime}(z)=\frac{g(z)}{f(z)}=\frac{\sum_{0}^{\infty} b_{i} z^{i}}{\sum_{0}^{\infty} a_{i} z^{i}},
$$

with $e^{V(z)}$ decaying to 0 fast enough at the boundary of its support. Let

$$
\begin{aligned}
& F(z)=e^{V(z)} I_{E}(z), \\
& \left.F(y, z)=e^{V(y)+V(z)} \operatorname{sg}(z-y)\right) I_{E}(y) I_{E}(z),
\end{aligned}
$$

be the corresponding weight functions. Comparing (2.1), (4.3) and (4.4) we find that

$$
\begin{aligned}
\hat{\tau}_{2 n}^{E}(t)=\frac{(2 n) ! c_{2 n}}{2^{n} n !} \sigma\left(\left(\iint_{\mathbb{R}^{2}} \psi^{+}(z) \psi^{+}(y) F(y, z) d y d z\right)^{n}|0\rangle\right), \\
\hat{\tau}_{2 n+1}^{E}(t)=\frac{(2 n) ! c_{2 n}}{2^{n} n !} \sigma\left(\iint_{\mathbb{R}^{2}} \psi^{+}(z) \psi^{+}(y) F(y, z) d y d z\right)^{n} \\
\left.\times\left(\int_{\mathbb{R}} \psi^{+}(w) F(w) d w\right)|0\rangle\right) .
\end{aligned}
$$

Consider the Virasoro algebra (see (3.7)) $L_{k}:=L_{k}^{1}, k \in \mathbb{Z}$, with central charge -2 . From the commutation relations (3.8) we deduce

$$
\left[L_{k}, \psi^{+}(z)\right]=\partial_{z}\left(z^{k+1} \psi^{+}(z)\right) .
$$

Hence,

$$
\begin{aligned}
& {\left[\sum_{\ell=0}^{\infty} a_{\ell} L_{k+\ell}, \iint_{\mathbb{R}^{2}} \psi^{+}(z) \psi^{+}(y) F(y, z) d y d z\right]} \\
& \quad=\sum_{\ell=0}^{\infty} a_{\ell} \iint_{\mathbb{R}^{2}}\left[L_{k+\ell}, \psi^{+}(z) \psi^{+}(y) F(y, z) d y d z\right] \\
& \quad=\sum_{\ell=0}^{\infty} a_{\ell} \iint_{\mathbb{R}^{2}} F(y, z)\left(\partial_{y} y^{k+\ell+1}+\partial_{z} z^{k+\ell+1}\right)\left(\psi^{+}(z) \psi^{+}(y)\right) d y d z \\
& \quad=\sum_{\ell=0}^{\infty} a_{\ell} \iint_{E^{2}}\left(\partial_{y} y^{k+\ell+1}+\partial_{z} z^{k+\ell+1}\right) \psi^{+}(z) \psi^{+}(y) e^{V(y)+V(z)} \operatorname{sg}(z-y) d y d z
\end{aligned}
$$




$$
\begin{aligned}
& +\sum_{\ell=0}^{\infty} a_{\ell} \iint_{E^{2}}\left(y^{k+\ell+1} V^{\prime}(y)+z^{k+\ell+1} V^{\prime}(z)\right) \psi^{+}(z) \psi^{+}(y) e^{V(y)+V(z)} \operatorname{sg}(y-z) d y d z \\
& +2 \sum_{\ell=0}^{\infty} a_{\ell} \iint_{E^{2}}\left(z^{k+\ell+1} \delta(z-y)-y^{k+\ell+1} \delta(z-y)\right) \psi^{+}(z) \psi^{+}(y) e^{V(y)+V(z)} d y d z
\end{aligned}
$$

where we have used $\partial_{z} \operatorname{sg}(z-y)=2 \delta(z-y)$. The last term on the right-hand side of (5.6) is equal to 0 , the first to

$$
\sum_{\ell=0}^{\infty} a_{\ell} \sum_{i=1}^{2 r} c_{i}^{k+\ell+1} \partial_{c_{i}}\left(\iint_{\mathbb{R}^{2}} \psi^{+}(z) \psi^{+}(y) F(y, z) d y d z\right)
$$

and the second to

$$
\begin{gathered}
-\sum_{\ell=0}^{\infty} b_{\ell} \iint_{E^{2}}\left(y^{k+\ell+1}+z^{k+\ell+1}\right) \psi^{+}(z) \psi^{+}(y) e^{V(y)+V(z)} \operatorname{sg}(z-y) d y d z \\
=-\sum_{\ell=0}^{\infty} b_{\ell} \iint_{\mathbb{R}^{2}}\left[\alpha_{k+\ell+1}, \psi^{+}(z) \psi^{+}(y)\right] F(y, z) d y d z \\
=-\sum_{\ell=0}^{\infty} b_{\ell}\left[\alpha_{k+\ell+1}, \iint_{\mathbb{R}^{2}} \psi^{+}(z) \psi^{+}(y) F(y, z) d y d z\right] .
\end{gathered}
$$

From which we conclude that

$$
\left[\sum_{\ell=0}^{\infty}\left(a_{\ell} \sum_{i=1}^{2 r} c_{i}^{k+\ell+1} \partial_{c_{i}}-a_{\ell} L_{k+\ell}-b_{\ell} \alpha_{k+\ell+1}\right), \iint_{\mathbb{R}^{2}} \psi^{+}(z) \psi^{+}(y) F(y, z) d y d z\right]=0 .
$$

An analogous calculation also shows that

$$
\left[\sum_{\ell=0}^{\infty}\left(a_{\ell} \sum_{i=1}^{2 r} c_{i}^{k+\ell+1} \partial_{c_{i}}-a_{\ell} L_{k+\ell}-b_{\ell} \alpha_{k+\ell+1}\right), \int_{\mathbb{R}} \psi^{+}(w) F(w) d w\right]=0 .
$$

Using the action of the Virasoro and oscillator algebra on the vacuum vector (3.9), one has that for all $k \geq-1$ and $m \geq 0$ :

$$
\sum_{\ell=0}^{\infty}\left(a_{\ell} \sum_{i=1}^{2 r} c_{i}^{k+\ell+1} \partial_{c_{i}}-a_{\ell} L_{k+\ell}-b_{\ell} \alpha_{k+\ell+1}\right)\left(\tau_{m}^{F}\right)=0 .
$$

Now recall from (3.10) that $W_{k}=-k t_{-k},=q \frac{\partial}{\partial q},=\frac{\partial}{\partial t_{k}}$ for $k<0, k=0, k>0$, respectively. Define

$$
W_{k}^{(2)}=\frac{1}{2} \delta_{\left[\frac{k}{2}\right], \frac{k}{2}} W_{\frac{k}{2}}^{2}+\sum_{i>\left[\frac{k}{2}\right]} W_{k-i} W_{i}+\frac{k+1}{2} W_{k},
$$

here $\left[\frac{N}{2}\right]=\operatorname{Entier}\left(\frac{N}{2}\right)$, and let as in Section 1

$$
\tau^{E}(t, q)=\sum_{m=0}^{\infty} \frac{\hat{\tau}_{m}^{E}(t)}{m ! c_{m}} q^{m},
$$

then using the boson-fermion correspondence we have the following result of Adler and van Moerbeke [4]: 
Proposition 5.1. For all $k \geq-1$ and all $m \geq 0$,

$$
\begin{aligned}
& \sum_{\ell=0}^{\infty}\left(a_{\ell} \sum_{i=1}^{2 r} c_{i}^{k+\ell+1} \partial_{c_{i}}-a_{\ell} W_{k+\ell}^{(2)}-b_{\ell} W_{k+\ell+1}\right)\left(\hat{\tau}_{m}^{E}(t) q^{m}\right)=0 \\
& \sum_{\ell=0}^{\infty}\left(a_{\ell} \sum_{i=1}^{2 r} c_{i}^{k+\ell+1} \partial_{c_{i}}-a_{\ell} W_{k+\ell}^{(2)}-b_{\ell} W_{k+\ell+1}\right)\left(\tau^{E}(t, q)\right)=0 .
\end{aligned}
$$

\section{$6 \quad$ Fay identity of the BKP hierarchy}

In this section we will prove the generalized Fay identity. We will use this identity to prove a recursion relation between certain pfaffian wave functions, which we will define in the next section.

Proposition 6.1. (Fay identity) Let $t-t^{\prime}=\sum_{k=1}^{p}\left[z_{k}\right]-\sum_{\ell=1}^{q}\left[y_{\ell}\right]$, where $[z]=\left(\frac{z}{1}, \frac{z}{2}, \frac{z}{3}, \ldots\right)$, then

$$
\begin{aligned}
& \frac{1}{2}\left(1-(-1)^{n+m}\right) \tau_{n}(t) \tau_{m}\left(t^{\prime}\right) \\
& =\sum_{k=1}^{p} z_{k}^{m-n+p-q} \tau_{n-1}\left(t-\left[z_{k}\right]\right) \tau_{m+1}\left(t^{\prime}+\left[z_{k}\right]\right) \frac{\prod_{i=1}^{q} z_{k}-y_{i}}{\prod_{j=1, j \neq k}^{p} z_{k}-z_{j}} \\
& +\left.\sum_{r=0}^{\infty} \frac{\delta_{n-m-p+q-1, r}}{r !}\left(\frac{\partial}{\partial z}\right)^{r}\left(\tau_{n-1}(t-[z]) \tau_{m+1}\left(t^{\prime}+[z]\right) \frac{\prod_{i=1}^{q} z-y_{i}}{\prod_{j=1}^{p} z-z_{j}}\right)\right|_{z=0} \\
& +\sum_{\ell=1}^{q} y_{\ell}^{n-m+q-p} \tau_{n+1}\left(t+\left[y_{\ell}\right]\right) \tau_{m-1}\left(t^{\prime}-\left[y_{\ell}\right]\right) \frac{\prod_{j=1}^{p} y_{\ell}-z_{p}}{\prod_{i=1, i \neq \ell}^{q} y_{\ell}-y_{i}} \\
& +\left.\sum_{r=0}^{\infty} \frac{\delta_{m-n+p-q-1, r}}{r !}\left(\frac{\partial}{\partial z}\right)^{r}\left(\tau_{n+1}(t+[z]) \tau_{m-1}\left(t^{\prime}-[z]\right) \frac{\prod_{j=1}^{p} z-z_{j}}{\prod_{i=1}^{q} z-y_{i}}\right)\right|_{z=0} .
\end{aligned}
$$

Proof. Notice that equation (1.4) is equal to

$$
\begin{aligned}
\frac{1}{2}(1- & \left.(-1)^{n+m}\right) \tau_{n}(t) \tau_{m}\left(t^{\prime}\right) \\
& =\frac{1}{2 \pi i} \oint_{z=0}\left(z^{n-m} \tau_{n-1}(t-[z]) \tau_{m+1}\left(t^{\prime}+[z]\right) e^{\xi\left(t, z^{-1}\right)-\xi\left(t^{\prime}, z^{-1}\right)}\right. \\
& \left.+z^{m-n} \tau_{n+1}(t+[z]) \tau_{m-1}\left(t^{\prime}[z]\right) e^{\xi\left(t^{\prime}, z^{-1}\right)-\xi\left(t, z^{-1}\right)}\right) d z
\end{aligned}
$$


where the integration is taken along a contour around $z=0$ and all $z=y_{k}$ and $z=z_{\ell}$. Since $t-t^{\prime}=\sum_{k=1}^{p}\left[z_{k}\right]-\sum_{\ell=1}^{q}\left[y_{\ell}\right]$, we find that

$$
e^{\xi\left(t, z^{-1}\right)-\xi\left(t^{\prime}, z^{-1}\right)}=z^{p-q} \frac{\prod_{i=1}^{q} z-y_{i}}{\prod_{j=1}^{p} z-z_{j}} .
$$

Hence the right-hand side of $(6.1)$ is equal to

$$
\begin{aligned}
\frac{1}{2 \pi i} \oint_{z=0}\left(z^{m-n+p-q} \tau_{n-1}(t-[z]) \tau_{m+1}\left(t^{\prime}+[z]\right) \frac{\prod_{i=1}^{q} z-y_{i}}{\prod_{j=1}^{p} z-z_{j}}\right. \\
\left.\quad+z^{n-m+q-p} \tau_{n+1}(t+[z]) \tau_{m-1}\left(t^{\prime}-[z]\right) \frac{\prod_{j=1}^{p} z-z_{j}}{\prod_{i=1}^{q} z-y_{i}}\right) d z .
\end{aligned}
$$

Now using the fact that the integrand has poles only at $z=0$ and all $z=y_{k}$ and $z=z_{\ell}$, we obtain the desired result.

As a particular case of Proposition 6.1 we take $n=N+1, m=N-2, p=1, z_{1}=u^{-1}$, $q=0, t^{\prime}=t-\left[u^{-1}\right]$ and observe that

$$
\left.\frac{\partial \tau(t \pm[z])}{\partial z}\right|_{z=0}= \pm \frac{\partial \tau(t \pm[z])}{\partial t_{1}}
$$

We thus obtain

\section{Corollary 6.1.}

$$
\begin{aligned}
& \tau_{N-1}(t) \tau_{N}\left(t-\left[u^{-1}\right]\right)=\tau_{N}(t) \tau_{N-1}\left(t-\left[u^{-1}\right]\right) \\
& \quad+u^{-1}\left(\tau_{N}(t) \frac{\partial \tau_{N-1}\left(t-\left[u^{-1}\right]\right)}{\partial t_{1}}-\tau_{N-1}\left(t-\left[u^{-1}\right]\right) \frac{\partial \tau_{N}(t)}{\partial t_{1}}\right) \\
& \quad+u^{-2} \tau_{N+1}(t) \tau_{N-2}\left(t-\left[u^{-1}\right]\right) .
\end{aligned}
$$

\section{Wave functions and skew orthogonal polynomials}

Introduce the following BKP wave functions:

$$
\Psi_{n}(t, z)=z^{n} P_{n}(t, z) e^{\xi(t, z)}:=z^{n} \frac{\tau_{n}\left(t-\left[z^{-1}\right]\right)}{\tau_{n+1}(t)} .
$$

These are different wave functions than the one described in [9] and [4]. Let $h_{n}(t)=$ $\frac{\left(\tau_{n+1}(t)\right)^{2}}{\tau_{n}(t) \tau_{n+2}(t)}$, using Corollary 6.1, one easily deduces the following recursion relation:

$$
\Psi_{n}(t, z)=h_{n-1}(t) \frac{\partial \Psi_{n-1}(t, z)}{\partial t_{1}}+\Psi_{n-2}(t, z) .
$$


From now on let $\tau_{n}(t)=\tau_{n}^{F}(t)=\operatorname{Pf}\left(M_{n}(t)\right)$ as in Section 4. It was shown in [1] that

$$
\begin{aligned}
& \tilde{P}_{2 n}(t, z):=P_{2 n}(t, z)
\end{aligned}
$$

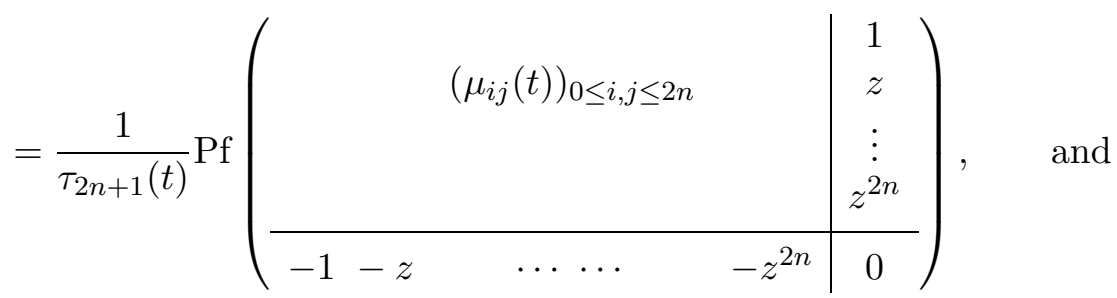

$$
\begin{aligned}
& \tilde{P}_{2 n+1}(t, z):=\frac{h_{2 n}(t)}{\tau_{2 n+1}(t)}\left(z+\frac{\partial}{\partial t_{1}}\right) \tau_{2 n+1} P_{2 n}(t, z)
\end{aligned}
$$

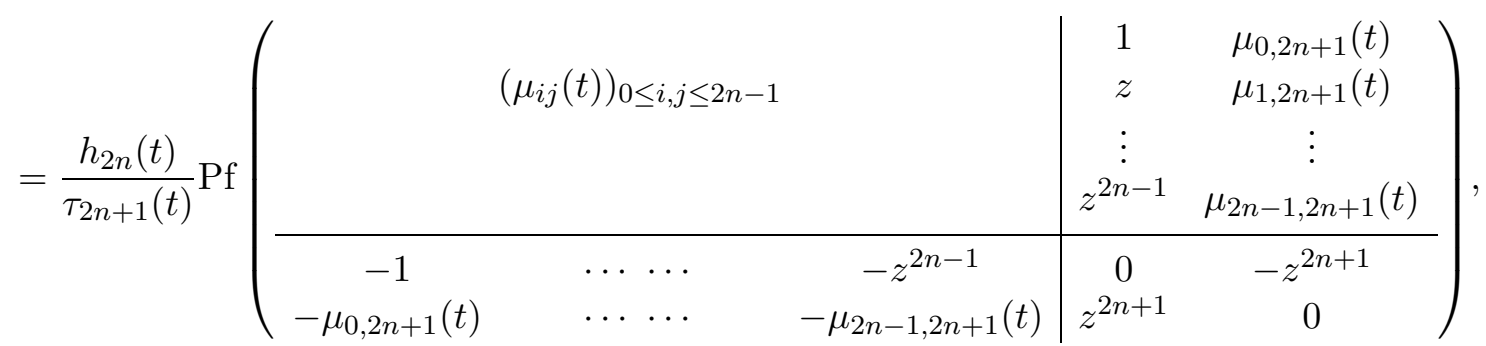

form a set skew orthonormal polynomials $\left\{\tilde{P}_{n}(t, z)\right\}_{n \geq 0}$ with respect to the time-dependent skew symmetric innerproduct $\langle\cdot, \cdot\rangle_{t}$, defined in (4.2), i.e.

$$
\begin{aligned}
& \left\langle\tilde{P}_{2 m}, \tilde{P}_{2 n}\right\rangle_{t}=\left\langle\tilde{P}_{2 m+1}, \tilde{P}_{2 n+1}\right\rangle_{t}=0, \\
& \left\langle\tilde{P}_{2 m}, \tilde{P}_{2 n+1}\right\rangle_{t}=-\left\langle\tilde{P}_{2 n+1}, \tilde{P}_{2 m}\right\rangle_{t}=\delta_{n m} .
\end{aligned}
$$

Now using (7.2), one sees that

$$
\tilde{P}_{2 n+1}(t, z)-h_{2 n}(t) \frac{\partial \log \tau_{2 n+1}(t)}{\partial t_{1}} \tilde{P}_{2 n}(t, z)=P_{2 n+1}(t)-P_{2 n-1}(t) .
$$

Let $Q_{2 n}(t, z)=P_{2 n}(t, z)$ and $Q_{2 n+1}(t, z)=P_{2 n+1}(t)-P_{2 n-1}(t)$, we thus have the following result:

Proposition 7.1. Let $\tau_{n}(t)=\tau_{n}^{F}(t)=\operatorname{Pf}\left(M_{n}(t)\right)$, then the polynomials

$$
\begin{aligned}
& Q_{2 n}(t, z):=z^{2 n} \frac{\tau_{2 n}\left(t-\left[z^{-1}\right]\right)}{\tau_{2 n+1}(t)}
\end{aligned}
$$

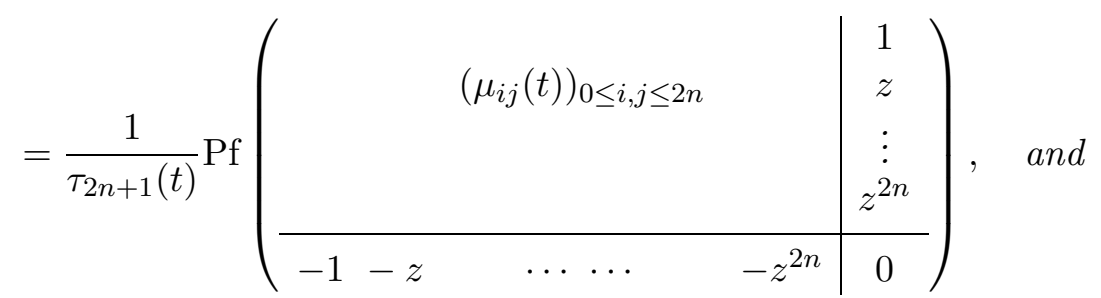




$$
\begin{aligned}
& Q_{2 n+1}(t, z):=z^{2 n+1} \frac{\tau_{2 n+1}\left(t-\left[z^{-1}\right]\right)}{\tau_{2 n+2}(t)}-z^{2 n-1} \frac{\tau_{2 n-1}\left(t-\left[z^{-1}\right]\right)}{\tau_{2 n}(t)}
\end{aligned}
$$

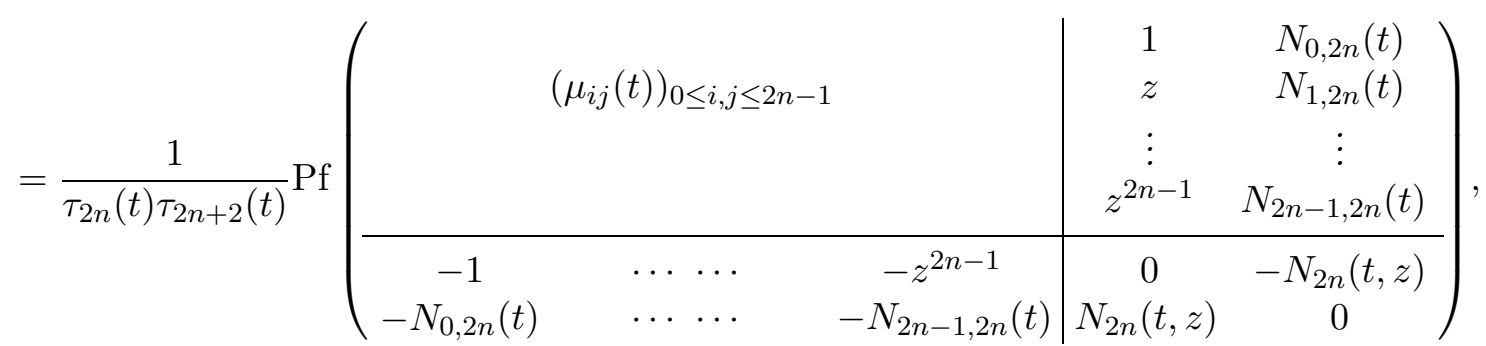

where

$$
\begin{aligned}
& N_{j, 2 n}(t)=\mu_{j, 2 n}(t) \frac{\partial \tau_{2 n+1}(t)}{\partial t_{1}}+\mu_{j, 2 n+1}(t) \tau_{2 n+1}(t), \quad \text { for } \quad 1 \leq j<2 n \\
& N_{2 n}(t, z)=z^{2 n} \frac{\partial \tau_{2 n+1}(t)}{\partial t_{1}}+z^{2 n+1} \tau_{2 n+1}(t)
\end{aligned}
$$

form a set skew orthonormal polynomials with respect to the time-dependent skew-symmetric innerproduct $\langle\cdot, \cdot\rangle_{t}$. Moreover,

$$
\begin{aligned}
& Q_{2 n+1}(t, z)=h_{2 n}(t)\left(z+\frac{\partial}{\partial t_{1}}\right) Q_{2 n}(t, z) \\
& Q_{2 n}(t, z)=\sum_{k=1}^{n}\left(\sum_{\ell=1}^{k} h_{2 n-2 \ell+1}\right)\left(z+\frac{\partial}{\partial t_{1}}\right) Q_{2 n-2 k+1}(t, z) .
\end{aligned}
$$

\section{Symplectic matrix integrals}

In this section we will treat the symplectic matrix integrals, i.e., integrals of the form:

$$
\int_{\mathcal{T}_{2 n}(E)} e^{2 \operatorname{Tr}\left(V(Z)+\sum_{1}^{\infty} t_{i} Z^{i}\right)} d Z
$$

where $d Z$ denotes the Haar measure

$$
d Z=\prod_{1}^{N} d Z_{k} \prod_{k \leq \ell} d Z_{k \ell}^{(0)} \overline{d Z_{k \ell}^{(0)}} d Z_{k \ell}^{(1)} \overline{d Z_{k \ell}^{(1)}},
$$

on the space $\mathcal{T}_{2 N}(E)$ of self-dual $N \times N$ Hermitean matrices with quaternionic entries and spectrum in $E \subset \mathbb{R}$; these particular matrices can be realized as the space of $2 N \times 2 N$ matrices with entries $Z_{\ell k}^{(i)} \in \mathbb{C}$

$$
\mathcal{T}_{2 N}=\left\{Z=\left(Z_{k \ell}\right)_{1 \leq k, \ell \leq N} \mid Z_{k \ell}=\left(\begin{array}{cc}
Z_{k \ell}^{(0)} & Z_{k \ell}^{(1)} \\
-\bar{Z}_{k \ell}^{(1)} & \bar{Z}_{k \ell}^{(0)}
\end{array}\right) \text { with } Z_{\ell k}=\bar{Z}_{k \ell}^{\top}\right\}
$$


It is shown in [5] that

$$
\begin{aligned}
\hat{\tau}_{2 n}^{E}(t) & =\int_{\mathcal{T}_{2 n}(E)} e^{2 \operatorname{Tr}\left(V(Z)+\sum_{1}^{\infty} t_{i} Z^{i}\right)} d Z \\
& =\int_{E^{n}} \Delta_{n}^{4}(z) \prod_{i=1}^{n}\left(e^{\left(V(z)+\sum_{1}^{\infty} t_{i} z^{i}\right)} d z_{i}\right) \\
& =n ! \operatorname{Pf}\left(\left(\mu_{i j}(t)\right)_{0 \leq i, j \leq 2 n-1}\right),
\end{aligned}
$$

where the $\mu_{i j}(t)$ are the moments of the skew-symmetric innerproduct

$$
\langle f, g\rangle_{t}=\int_{\mathbb{R}}\left(\frac{\partial f(z)}{\partial z} g(z)-\frac{\partial g(z)}{\partial z} f(z)\right) e^{2 \xi(t, z)} F(z) d z
$$

and $F(z)=e^{2 V(z)} I_{E}(z)$. We will now show that the generating series

$$
\tau^{E}(t, q)=\sum_{n=0}^{\infty} \tau_{2 n}^{E}(t) q^{2 n}, \quad \text { with } \quad \hat{\tau}_{2 n}^{E}(t)=n ! \tau_{2 n}^{E}(t)
$$

of these Pfaffians is again an element in the Spin group orbit of the vacuum vector.

Let $F(z)$ be a weight function on $\mathbb{R}$ and introduce the following symmetric, respectively skew-symmetric inner product:

$$
\begin{aligned}
& (f, g)=\int_{\mathbb{R}} f(z) g(z) F(z) d z, \quad \text { respectively } \\
& \langle f, g\rangle=\int_{\mathbb{R}}\left(\frac{\partial f(z)}{\partial z} g(z)-\frac{\partial g(z)}{\partial z} f(z)\right) F(z) d z
\end{aligned}
$$

with moments $\mu_{i}=\left(z^{i}, 1\right)$ respectively $\mu_{i j}=\left\langle z^{i}, z^{j}\right\rangle$. Notice that $\langle\cdot, \cdot\rangle_{t}$, given by (8.1), is a time-dependent $t=\left(t_{1}, t_{2}, \cdots\right)$ deformation of $\langle\cdot, \cdot\rangle$. In a similar way is

$$
(f, g)_{t}=\int_{\mathbb{R}} f(z) g(z) e^{2 \xi(t, z)} F(z) d z
$$

a the time-dependent deformation of $(\cdot, \cdot)$.

To describe this symplectic case, we consider the generalized Spin group element

$$
\begin{aligned}
H^{\mu} & =\exp \left(h^{\mu}\right)=\exp \left(\sum_{j<i<0} \mu_{-j-\frac{1}{2},-i-\frac{1}{2}} \psi_{j}^{+} \psi_{i}^{+}\right) \\
& =\exp \left(\sum_{j<i<0}(i-j) \mu_{-i-j-2} \psi_{j}^{+} \psi_{i}^{+}\right)=\exp \left(\sum_{i, j<0}\left(-j-\frac{1}{2}\right) \mu_{-i-j-2} \psi_{j}^{+} \psi_{i}^{+}\right)
\end{aligned}
$$


and calculate its action on the vacuum vector:

$$
\begin{aligned}
\tau^{F} & =\exp \left(\sum_{j<i<0} \mu_{-j-\frac{1}{2},-i-\frac{1}{2}} \psi_{j}^{+} \psi_{i}^{+}\right)|0\rangle \\
& =\sum_{n=0}^{\infty} \frac{1}{n !}\left(\sum_{i, j<0}\left(-j-\frac{1}{2}\right) \mu_{-i-j-2} \psi_{j}^{+} \psi_{i}^{+}\right)^{n}|0\rangle \\
& =\sum_{n=0}^{\infty} \frac{1}{n !}\left(\sum_{i, j<0} \int_{\mathbb{R}} z^{-i-j-2} F(z) d z\left(-j-\frac{1}{2}\right) \psi_{j}^{+} \psi_{i}^{+}\right)^{n}|0\rangle \\
& =\sum_{n=0}^{\infty} \frac{1}{n !}\left(\int_{\mathbb{R}} Y^{+}(z) F(z) d z\right)^{n}|0\rangle \\
& =\sum_{n=0}^{\infty} \frac{1}{n !} \int_{\mathbb{R}^{n}} Y^{+}\left(z_{n-1}\right) Y^{+}\left(z_{n-2}\right) \cdots Y^{+}\left(z_{0}\right) \prod_{j=0}^{n-1} F\left(z_{j}\right) d z_{j}|0\rangle \\
& =\sum_{n=0}^{\infty} \tau_{2 n}^{F} .
\end{aligned}
$$

Use again the boson-fermion correspondence and corollary 3.1. Let $\tau^{F}(t, q)=\sigma\left(\tau^{F}\right)$, then

$$
\tau^{F}(t, q)=\sum_{n=0}^{\infty} \frac{1}{n !} \int_{\mathbb{R}^{n}} \Delta_{n}^{4}(z) \prod_{j=0}^{n-1} e^{2 \xi\left(t, z_{j}\right)} F\left(z_{j}\right) d z_{j} q^{2 n}=\sum_{n=0}^{\infty} \tau_{2 n}^{F}(t) q^{2 n}
$$

Let as in Section $5 E$ satisfy (5.1) and assume that $F(z)=e^{2 V(z) I_{E}(z)}$, where $V(z)$ satisfies (5.2). Let $L(z)=L^{0}(z)$ (c.f. (3.7) ) be the Virasoro field. Although this field has central charge -2 , note that it is another Virasoro field than the one one considers in the symmetric case. In a similar way as Section 5 one shows that

$$
\left[\sum_{\ell=0}^{\infty}\left(a_{\ell} \sum_{i=1}^{2 r} c_{i}^{k+\ell+1} \partial_{c_{i}}-a_{\ell} L_{k+\ell}-b_{\ell} \alpha_{k+\ell+1}\right), \int_{\mathbb{R}} Y^{+}(w) F(w) d w\right]=0 .
$$

Which leeds to the following result.

Theorem 8.1. Let $\mu_{i j}(t)$ be the moments of the skew-symmetric inner product (see (8.1)) $\langle\cdot, \cdot\rangle_{t}$ with weight function $F(z)=e^{2 V(z)} I_{E}(z)$ and let

$$
Y(t, z)=q^{2} z^{2 q \frac{\partial}{\partial q}} \exp \left(2 \sum_{i=1}^{\infty} t_{i} z^{i}\right) \exp \left(-2 \sum_{i=1}^{\infty} \frac{z^{-i}}{i} \frac{\partial}{\partial t_{i}}\right),
$$

then

$$
\begin{aligned}
& \sum_{n=0}^{\infty} \frac{1}{n !} \hat{\tau}_{2 n}^{E}(t) q^{2 n}=\sigma\left(\exp \left(\sum_{j<i<0} \mu_{-j-\frac{1}{2},-i-\frac{1}{2}}(0) \psi_{j}^{+} \psi_{i}^{+}\right)|0\rangle\right) \\
& \quad=\exp \left(\int_{\mathbb{R}} Y(t, z) F(z) d z\right) \cdot 1 .
\end{aligned}
$$


All $\tau_{2 n}^{E}(t)$ satisfy the DKP hierarchy, i.e., the BKP hierarchy but only for the even taufunctions. Moreover, let

$$
W_{k}^{(2)}=\frac{1}{2} \delta_{\left[\frac{k}{2}\right], \frac{k}{2}} W_{\frac{k}{2}}^{2}+\sum_{i>\left[\frac{k}{2}\right]} W_{k-i} W_{i}-\frac{k+1}{2} W_{k},
$$

then for all $k \geq-1$ and all $n \geq 0$,

$$
\sum_{\ell=0}^{\infty}\left(a_{\ell} \sum_{i=1}^{2 r} c_{i}^{k+\ell+1} \partial_{c_{i}}-a_{\ell} W_{k+\ell}^{(2)}-b_{\ell} W_{k+\ell+1}\right)\left(\hat{\tau}_{2 n}^{E}(t) q^{2 n}\right)=0 .
$$

\section{Consequences of the Virasoro constraints}

In this section we will describe some consequences of the Virasoro constraints. First consider the following. Let $\tau=g|0\rangle, u \in$ Ann $\tau$ and let $A$ be an operator, such that $[A, V] \subset V$ and $A \tau=0$, then

$$
0=A u \tau=[A, u] \tau+u A \tau=[A, u] \tau
$$

and thus $[A, u] \in \operatorname{Ann} \tau$. The operators $(k \geq-1)$

$$
A_{k}:=\sum_{\ell=0}^{\infty}\left(a_{\ell} \sum_{i=1}^{2 r} c_{i}^{k+\ell+1} \partial_{c_{i}}-a_{\ell} L_{k+\ell}-b_{\ell} \alpha_{k+\ell+1}\right)
$$

satisfy this condition for $\tau=\tau^{F}$ in both symmetric and symplectic cases and for $\tau^{g}:=$ $\sum_{m=0}^{\infty} \tau_{2 m}^{F} q^{2 m}=\exp \left(g^{\mu}\right)|0\rangle$ in the symmetric case.

First, we consider the symmetric case. We calculate Ann $\tau^{F}=T_{G^{\mu}}($ Ann $|0\rangle)=$ $T_{G^{\mu}}\left(U_{0}\right)$. Since for all $k>0$ and all $\ell$

$$
T_{f^{\mu}}\left(\psi_{k}^{-}\right)=\psi_{k}^{-}+\sqrt{2} \mu_{k-\frac{1}{2}} \psi_{0}-\mu_{k-\frac{1}{2}} \sum_{i<0} \mu_{-i-\frac{1}{2}} \psi_{i}^{+},
$$

$T_{f^{\mu}}\left(\psi_{k}^{+}\right)=\psi_{k}^{+},\left[g^{\mu}, \psi_{\ell}^{+}\right]=\left[g^{\mu}, \psi_{0}\right]=0$ and $\left[g^{\mu}, \psi_{k}^{-}\right]=\sum_{i<0} \mu_{k-\frac{1}{2},-i-\frac{1}{2}} \psi_{i}^{+}$, we find that

$$
\text { Ann } \tau^{F}=\sum_{k>0} \mathbb{C} \psi_{k}^{+}+\mathbb{C} \Psi_{k}^{-}
$$

with

$$
\Psi_{k}^{-}=\psi_{k}^{-}+\sqrt{2} \mu_{k-\frac{1}{2}} \psi_{0}+\sum_{i>0}\left(\mu_{k-\frac{1}{2}, i-\frac{1}{2}}-\mu_{k-\frac{1}{2}} \mu_{i-\frac{1}{2}}\right) \psi_{-i}^{+} .
$$

In simmilar way one obtains

$$
\text { Ann } \tau^{g}=\sum_{k>0} \mathbb{C} \psi_{k}^{+}+\mathbb{C} \Phi_{k}^{-}
$$


with

$$
\Phi_{k}^{-}=\psi_{k}^{-}+\sum_{i>0} \mu_{k-\frac{1}{2}, i-\frac{1}{2}} \psi_{-i}^{+}
$$

In the symmetric case we have:

$$
\left[\alpha_{k}, \psi_{i}^{ \pm}\right]= \pm \psi_{i+k}^{ \pm}, \quad\left[L_{k}, \psi_{i}^{ \pm}\right]=-i-\frac{k}{2} \pm \frac{k+1}{2} .
$$

Since,

$$
\left[A_{k}, \psi_{j}^{-}\right]=\sum_{\ell=0}\left(j+k+\ell+\frac{1}{2}\right) a_{\ell} \psi_{j+k+\ell}^{-}+b_{\ell} \psi_{j+k+\ell+1},
$$

one has that

$$
\begin{aligned}
{\left[A_{k}, \Psi_{j}^{-}\right]=} & \sum_{\ell=0}\left(j+k+\ell+\frac{1}{2}\right) a_{\ell} \Psi_{j+k+\ell}^{-}+b_{\ell} \Psi_{j+k+\ell+1} \\
& + \text { part which contains only } \psi_{i}^{+} \text {'s with } i>0 .
\end{aligned}
$$

Now comparing the coefficient on both sides of $\psi_{0}$, we deduce the following equation for all $k \geq-1$ :

$$
\sum_{\ell=0}^{\infty} a_{\ell} \sum_{p=1}^{2 r} c_{p}^{k+\ell+1} \frac{\partial \mu_{j}}{\partial c_{p}}=\sum_{\ell=0}^{\infty}(j+k+\ell+1) a_{\ell} \mu_{j+k+\ell}+b_{\ell} \mu_{j+k+\ell+1} .
$$

The same equation $(9.1)$ holds with $\Psi_{j}^{-}$replaced by $\Phi_{j}^{-}$. Now consider the coefficient of $\psi_{-i}^{+}$for $i>0$ on both sides. This leads to:

$$
\begin{aligned}
\sum_{\ell=0}^{\infty} a_{\ell} \sum_{p=1}^{2 r} c_{p}^{k+\ell+1} \frac{\partial \mu_{j, i}}{\partial c_{p}}= & \sum_{\ell=0}^{\infty} a_{\ell}\left((i+k+\ell+1) \mu_{j, i+k+\ell}+(j+k+\ell+1) \mu_{j+k+\ell, i}\right) \\
& +b_{\ell}\left(\mu_{j, i+k+\ell+1}+\mu_{j+k+\ell+1, i}\right) .
\end{aligned}
$$

In the symplectic case we find:

$$
\text { Ann } \tau^{F}=\sum_{k>0} \mathbb{C} \psi_{k}^{+}+\mathbb{C} \Theta_{k}^{-},
$$

with

$$
\Theta_{k}^{-}=\psi_{k}^{-}+\sum_{i>0}(i-k) \mu_{k+i-2} \psi_{-i}^{+}
$$

In this case

$$
\left[\alpha_{k}, \psi_{i}^{ \pm}\right]= \pm \psi_{i+k}^{ \pm}, \quad\left[L_{k}, \psi_{i}^{ \pm}\right]=-i-\frac{k}{2} \mp \frac{k+1}{2},
$$

which leads to the following result for the symplectic case:

$$
\sum_{\ell=0}^{\infty} a_{\ell} \sum_{p=1}^{2 r} c_{p}^{k+\ell+1} \frac{\partial \mu_{j}}{\partial c_{p}}=\sum_{\ell=0}^{\infty}(j+k+\ell+1) a_{\ell} \mu_{j+k+\ell}+2 b_{\ell} \mu_{j+k+\ell+1}
$$

and

$$
\sum_{\ell=0}^{\infty} a_{\ell} \sum_{p=1}^{2 r} c_{p}^{k+\ell+1} \frac{\partial \mu_{j, i}}{\partial c_{p}}=\sum_{\ell=0}^{\infty} a_{\ell}\left(i \mu_{j, i+k+\ell}+j \mu_{j+k+\ell, i}\right)+b_{\ell}\left(\mu_{j, i+k+\ell+1}+\mu_{j+k+\ell+1, i}\right) .
$$




\section{References}

[1] Adler M, Horozov E and van Moerbeke P, The Pfaff Lattice and Skew-Orthogonal Polynomials, Internat. Math. Res. Notices 11 (1999), 569-588.

[2] Adler M, Shiota T and van Moerbeke P, Pfaff $\tau$-functions, solv-int/9909010.

[3] Adler M and van Moerbeke P, The Spectrum of Coupled Random Matrices, Annals of Math. 149 (1999), 921-976.

[4] Adler M and van Moerbeke P, Symmetric Random Matrices and the Pfaff Lattice, solvint/9903009.

[5] Adler M and van Moerbeke P, The Pfaff Lattice, Matrix Integrals and a Map from Toda to Pfaff, preprint.

[6] Date E, Jimbo M, Kashiwara M and Miwa T, Operator Approach to the KadomtsevPetviashvili Equation. Transformation Groups for Soliton Equations. III, J. Phys. Soc. Japan 50 (1981), 3806-3812.

[7] Jimbo M and Miwa T, Solitons and Infinite Dimensional Lie Algebras, Publ. Res. Inst. Math. Sci. 19 (1983), 943-1001.

[8] Kac V G, Vertex Algebras for Beginners, University Lecture Series 10, AMS, Providence, RI, 1996.

[9] Kac V G and van de Leur J W, The Geometry of Spinors and the Multicomponent BKP and DKP Hierarchies, CRM Proceedings and Lecture Notes 14, 1998, 159-202.

[10] Kac V G and Raina A K, Bombay Lectures on Highest Weight Representations of InfiniteDimensional Lie Algebras, Advanced Ser. in Math. Phys., Vol. 2, World Scientific 1987.

[11] Peng H, The Spectrum of Random Matrices for Symmetric Ensembles, Brandeis Dissertation, 1997.

[12] Ueno K and Takasaki K, Toda Lattice Hierarchy, Adv. Studies in Pure Math. 4 (1984), 1-95. 